\title{
A INFLUÊNCIA DA GINÁSTICA LABORAL NOS DISTÚRBIOS OSTEOMUSCULARES RELACIONADOS AO TRABALHO
}

\author{
THE INFLUENCE OF LABOR GYMNASTICS IN OSTEOMUSCULARS DISORDERS \\ RELATED TO WORK
}

\begin{abstract}
Juliana de Oliveira Souza ${ }^{a \star}$, Marcela Teixeira Matin ${ }^{b * *}$, Carine Muniz de Souza ${ }^{c *}$, Isabela Coelho Baptista $^{\mathrm{d}^{* *}}$, Lauane Pereira Cardoso ${ }^{\mathrm{e}^{* \star}}$, Geisiane Braz de Medeiros ${ }^{\mathrm{f} *}$, Ariela Torres $\mathrm{Cruz}^{\mathrm{g}^{* * *}}$, Priscila de Oliveira Januário ${ }^{\mathrm{h}^{* * *}}$

jufisio2011@hotmail.com ${ }^{\mathrm{a}}$, maarcela_martins@hotmail.com ${ }^{\mathrm{b}}$,cmsouza-28@hotmail.com ${ }^{\mathrm{c}}$, isabela.cbap@gmail.com ${ }^{\mathrm{d}}$, lauane.fisioterapia@gmail.com ${ }^{\mathrm{e}}$, geisi.bm@hotmail.com ${ }^{\mathrm{f}}$, ariela_tcruz@yahoo.com.br ${ }^{\mathrm{g}}$, pri.januario@gmail.com ${ }^{\mathrm{h}}$

Faculdade Unyleya - Rio de Janeiro, RJ ${ }^{*}$, Centro Universitário de Barra Mansa - UBM ${ }^{*}$, Universidade do Vale do Paraíba - Univap ${ }^{\star * *}$
\end{abstract}

Data do recebimento do artigo: 29/07/2018

Data do aceite: 08/10/2018

\section{RESUMO}

Introdução: A ginástica laboral (GL) são exercícios diários que tem o intuido de promover benefícios à saúde dos trabalhadores. Objetivos: Analisar os efeitos da GL nos distúrbios osteomusculares relacionados ao trabalho em funcionários de um setor administrativo do ramo siderúrgico. Materiais e Métodos: Participaram deste estudo 31 funcionários, divididos em dois grupos: grupo experimental ( $\mathrm{GE} n=16$ ) e grupo controle (GC $\mathrm{n}=15$ ). Ambos foram avaliados antes e após as sessões de GL através do Questionário Nórdico de Sintomas Osteomusculares. Após a avaliação, os funcionários do GE foram submetidos a 16 sessões de GL, realizadas 2 vezes por semana, com duração média de 15 minutos. Resultados: No GE houve uma diminuição das queixas de dor/formigamento principalmente na parte superior das costas, enquanto no GC houve um aumento dessas queixas na parte inferior das costas e nos punhos/mãos. Quanto à realização das atividades laborativas, domésticas e de lazer observou-se no GE uma diminuição das queixas na parte inferior das costas, enquanto houve um aumento no GC. Em ambos os grupos houve diminuição na procura por médico/fisioterapeuta, principalmente por problemas na parte inferior das costas. No GE observou-se redução das queixas por algum problema principalmente na parte inferior das costas, porém no GC houve aumento das queixas nesta região. Conclusões: As sessões de GL mostraram-se eficazes sobre os distúrbios osteomusculares dos funcionários do GE, diminuindo os relatos de queixas de dor/formigamento para a realização das atividades laborativas, domésticas e de lazer, bem como diminuição das consultas com profissionais de saúde.

Palavras-chave: Saúde do trabalhador; promoção da saúde; LER-DORT

\section{ABSTRACT}

Introduction: Labor gymnastics (LG) are daily exercises that have the intuition of promoting health benefits for workers. Objectives: To analyze the effects of LG in work-related osteomuscular disorders in employees of an administrative sector in the steel industry. Materials and methods: Thirty-one employees participated in this study, divided into two groups: experimental group $(E G \mathrm{n}=16)$ and control group $(\mathrm{CG} n=15)$. Both were evaluated before and after LG sessions through the Nordic Osteomuscular Symptoms Questionnaire. After the evaluation, EG employees were submitted to 16LG sessions, performed twice a week, with an average duration of 15 minutes. Results: In EG there was a decrease in pain / tingling complaints mainly in the upper back, while in CG there was an increase in these complaints in the lower back and wrists / hands. Regarding the performance of work, domestic and leisure activities, there was a decrease in complaints in the lower back, while there was an increase in CG. In both groups there was a decrease in the demand for a physician / physiotherapist, mainly due to problems in the lower back. In EG, there was a reduction in complaints due to some problem, mainly in the lower back, but in CG there was an increase in complaints in this region. Conclusions: The LG sessions are effective on the osteomuscular disorders of the employees of the EG, reducing the reports of complaints of pain / tingling to perform work, domestic and leisure activities, as well as decreasing consultations with health professionals.

Keywords: Occupational health; health promotion; RSI-WMSD 


\section{INTRODUÇÃO}

Atualmente, o trabalho vem ocupando um espaço muito importante na vida de qualquer indivíduo, e o excesso de atividades laborais, na maioria das vezes, é visto como o principal causador de males físicos e mentais. As transformações no mundo do trabalho têm ocorrido de forma rápida, impondo novas exigências aos trabalhadores, como novas formas de contratos e modos de organização do trabalho, que podem repercutir na saúde desses trabalhadores e nos processos de adoecimento e de retorno ao trabalho ${ }^{1}$.

Dentre algumas formas de adoecimento relacionado ao trabalho estão as Lesões por Esforços Repetitivos (LER) e Distúrbios Osteomusculares Relacionados ao Trabalho (DORT). Esses distúrbios osteomusculares incluem uma variedade de condições inflamatórias e degenerativas que afetam os músculos, tendões, ligamentos, articulações e nervos periféricos. São caracterizados pela ocorrência de vários sintomas, tais como dor, parestesia, sensação de peso e fadiga, que podem levar a incapacidade laboral, temporária ou permanente ${ }^{2}$.

Estudos têm mostrado a necessidade da implantação da Ginástica Laboral (GL) para a prevenção de patologias ocupacionais, para diminuição de acidentes e do absenteísmo no trabalho, assim como a queda dos gastos com assistência médica e aumento na produtividade da empresa ${ }^{4}$. A GL consiste em exercícios praticados diariamente em algumas empresas, podendo ser classificada de acordo com o seu horário de execução: a preparatória, realizada no início da jornada de trabalho com o objetivo de despertar diversos grupos musculares; a compensatória, praticada no meio do expediente com objetivo de exercícios específicos para esforços repetitivos ou estruturas sobrecarregadas e a de relaxamento, realizada após a jornada de trabalho ${ }^{5,6}$.

Os trabalhadores não são os únicos beneficiados pela introdução da GL no ambiente de trabalho. Porém muitas empresas não possuem esse modo de analisar e insistem em continuar com a forma atual de trabalho que leva o colaborador a fadiga mental e corporal, dor, estresse e acidentes de trabalho que demandam muito gasto e que poderiam ser minimizadas por técnicas de atividades coordenadas por equipes especializadas em saúde e qualidade de vida do trabalhador7. Um programa de GL visa combater e prevenir que a rotina do trabalho, o modo como é feito e a forma como ele é cobrado por superiores não afete a saúde do funcionário e que sua estabilidade e rentabilidade sejam sempre fatores que contribuam positivamente ao objetivo da empresa ${ }^{8}$.

Assim, as atividades de GL são sempre constituídas de práticas diversificadas e elaboradas para que os funcionários se sintam motivados para realizarem as ações. O tempo de parada na jornada de trabalho é analisado e são feitos os procedimentos que corroboram ao benefício do colaborador, dentre eles: exercícios de alongamentos, relaxamentos, exercícios ativos livres, resultando no despertar dos trabalhadores para continuar suas funções no trabalho9. Somado aos benefícios diretos da realização correta e contínua da GL é constatado o estímulo para uma alteração no estilo de vida com a inclusão de exercícios físicos no horário de lazer/descanso dos trabalhadores, uma reeducação alimentar e uma consciência sobre a postura no momento da realização do trabalho ${ }^{10}$.

Diante do exposto, e tendo como base a premissa que passamos grande parte de nossas vidas no trabalho, este estudo teve por objetivo geral analisar os efeitos da GL nos distúrbios osteomusculares relacionados ao trabalho em funcionários de um setor administrativo do ramo siderúrgico.

\section{METODOLOGIA}

Trata-se de um estudo de caráter observacional, descritivo e quantitativo, que teve início após a aprovação pelo Comitê de Ética em Pesquisa do Centro Universitário de Barra Mansa (UBM), sob o CAAE de no 59697616.2.0000.5236, respeitando todos os princípios éticos que norteiam a pesquisa, bem como a privacidade de seus conteúdos, como preconizam os documentos internacionais e a Resolução 466/12 do Conselho Nacional de Saúde do Ministério da Saúde.

Participaram deste estudo 31 funcionários, de ambos os gêneros, com idade entre 18 a 60 anos, que foram escolhidos aleatoriamente em uma empresa com atuação no ramo siderúrgico, localizada em um município do Médio Paraíba - RJ 
e que aceitaram fazer parte do estudo conforme o Termo de Consentimento Livre e Esclarecido (TCLE), explicando todo o procedimento metodológico, objetivos, riscos, benefícios e instrumentos de avaliação.

Os critérios de inclusão foram: trabalhadores com tempo institucional de no mínimo um ano e que não tivessem qualquer impedimento físico ou mental por justificativa médica para participarem das atividades durante a coleta dos dados; os que concordassem em assinar o TCLE e fossem assíduos nas sessões de GL, sem faltas consecutivas.

Os critérios de exclusão foram: funcionários (as) sem consentimento imediato da chefia para a realização da GL; portadores de deficiência física; em período gestacional, aqueles que estivessem realizando outros tratamentos para distúrbios osteomusculares simultaneamente, que fossem afastados da empresa ou precisassem abandonar o estudo por férias.

Os funcionários foram divididos em dois grupos: grupo experimental (GE), submetidas às sessões de GL; e grupo controle (GC), que não receberam a intervenção proposta. No próprio ambiente de trabalho, ambos os grupos foram avaliados antes e após as sessões de GL através do Questionário Nórdico de Sintomas Osteomusculares, traduzido e validado por Kuorinka et al., $(1987)^{11}$. O instrumento utilizado contém quatro perguntas fechadas sobre nove áreas corporais que visam identificar possíveis problemas musculoesqueléticos. As perguntas eram relacionadas à dor/ formigamento; as atividades laborativas; domésticas e de lazer; consultas ao médico ou fisioterapeuta e presença de algum problema nas regiões do pescoço, ombros, parte superior das costas, cotovelos, punhos/mãos, parte inferior das costas, quadril/coxas, joelhos e tornozelos/pés.

Após a avaliação, os funcionários do GE foram submetidos a 16 sessões de GL, realizadas 2 vezes por semana, com duração média de 15 minutos. O protocolo de GL foi elaborado pelas próprias pesquisadoras baseado na literatura e direcionado para os grupos musculares mais requisitados pelos funcionários durante as atividades laborais. Os funcionários do GE realizaram 16 sessões de GL em posição ortostática de forma preparatória e compensatória, realizadas 2 vezes por semana, com duração média de 15 minutos. O protocolo constava de exercícios de relaxamento, alongamento, mobilização ativa e exercícios respiratórios. Os exercícios de alongamentos foram realizados em 3 séries de 10 segundos e os exercícios ativos em 2 séries de 10 repetições, todos realizados de forma bilateral. Ao término de cada sessão foram realizados para o relaxamento, exercícios respiratórios sendo 1 série de 5 repetições. Foram analisados os períodos antes e após as 16 sessões de GL. Cabe ressaltar que, a seleção dos participantes, a aplicação do questionário e as sessões de GL foram realizadas pelo mesmo pesquisador previamente treinado.

Para análise dos dados coletados foram utilizados métodos estatísticos simples no programa Microsoft Excel, através de cálculos percentuais expressos na forma de gráficos e tabelas.

\section{RESULTADOS}

Os 45 funcionários do setor administrativo da empresa aceitaram fazer parte desta pesquisa. Dentre eles, 31,1\% (14 funcionários) não puderam participar, pois se enquadraram nos critérios de exclusão. O GE foi composto por 25 funcionários, sendo que 9 foram excluídos por motivos como afastamento, férias e por não participarem assiduamente das sessões de GL, totalizando assim 16 indivíduos. O GC foi composto por 20 funcionários, sendo que 6 foram excluídos por motivos como afastamento e férias, resultando em 15 indivíduos.

Após a aplicação do Questionário Nórdico de Sintomas Osteomusculares foi possível observar diminuição dos sintomas de dor/formigamento no GE entre os períodos antes e após as 16 sessões de GL nos segmentos de pescoço (de 56,2\% para $37,5 \%$ ); ombros (de $43,7 \%$ para $12,5 \%$ ); parte superior das costas (de $56,5 \%$ para $31,2 \%$ ); punhos e mãos (de $37,5 \%$ para $12,5 \%$ ); parte inferior das costas (de 50,0\% para $31,2 \%$ ); quadril/coxas (de $37,5 \%$ para $6,2 \%$ ); joelhos (de $25 \%$ para $18,7 \%$ ) e tornozelos/pés (de 18,7\% para 6,2\%). Mesmo após a realização da GL os sintomas mantiveram-se no segmento de cotovelo $(6,2 \%)$ entre os períodos estudados. Os dados podem ser observados na Tabela 1 juntos com a média dos intervalos e o desvio padrão. 
Tabela 1: Queixas de dor/formigamento dos funcionários do GE.

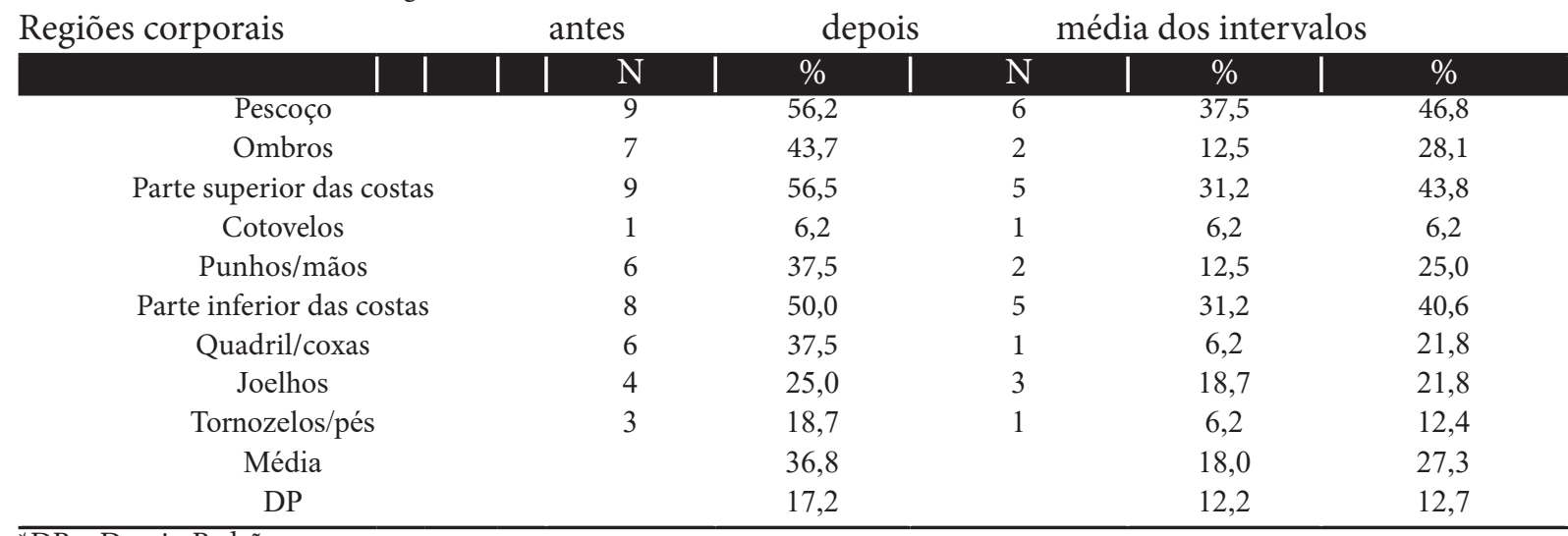

${ }^{\star}$ DP - Desvio Padrão

Já no GC, foi possível observar o aumento dos sintomas de dor/ formigamento entre os períodos estudados nos segmentos de pescoço (de 33,3\% para $46,4 \%$ ); ombros (de $26,6 \%$ para $33,3 \%$ ); parte superior das costas (de $20,0 \%$ para $46,4 \%$ ); punhos/mãos (de $40,0 \%$ para $46,4 \%$ ); parte infe- rior das costas (de $40,0 \%$ para $46,4 \%$ ); quadril/ coxas (de $13,3 \%$ para $20,0 \%$ ), a presença de dor nos tornozelos/pés (40\%) e nos cotovelos $(6,6 \%)$ manteve-se entre os períodos estudados. Os dados podem ser observados na Tabela 2 juntos com a média dos intervalos e o desvio padrão.

Tabela 2: Queixas de dor/formigamento dos funcionários do GC.

\begin{tabular}{|c|c|c|c|c|c|}
\hline & & & & & \\
\hline & $\mathbf{N}$ & $\%$ & $\mathbf{N}$ & $\%$ & $\%$ \\
\hline Pescoço & 5 & 33,3 & 7 & 46,4 & 39,8 \\
\hline Ombros & 4 & 26,6 & 5 & 33,3 & 29,9 \\
\hline Parte superior das costas & 3 & 20,0 & 7 & 46,4 & 33,2 \\
\hline Cotovelos & 1 & 6,6 & 1 & 6,6 & 6,6 \\
\hline Punhos/mãos & 6 & 40,0 & 7 & 46,4 & 43,2 \\
\hline Parte inferior das costas & 6 & 40,0 & 7 & 46,4 & 43,2 \\
\hline Quadril/coxas & 2 & 13,3 & 3 & 20,0 & 16,6 \\
\hline Joelhos & 7 & 46,6 & 6 & 40,0 & 43,3 \\
\hline Tornozelos/pés & 6 & 40,0 & 6 & 40,0 & 40,0 \\
\hline Média & & 29,6 & & 36,1 & 32,8 \\
\hline DP & & 13,7 & & 14,1 & 13,1 \\
\hline
\end{tabular}

Verificou-se diminuição dos relatos de queixas para a realização das atividades laborativas, domésticas e de lazer no grupo GE entre os períodos antes e após 16 sessões de GL nos segmentos ombros (de $12,5 \%$ para 6,2\%); parte superior das costas (de $18,7 \%$ para $12,5 \%$ ); cotovelos (de $0 \%$ para 6,2\%); parte inferior das costas (de $31,2 \%$ para $18,7 \%$ ); quadril/coxas (de $6,2 \%$ para
$0 \%$ ); joelhos (de $12,5 \%$ para $6,2 \%$ ) e tornozelos/ pés (de $6,2 \%$ para $0 \%$ ). Mesmo após a realização da GL as queixas mantiveram-se nos segmentos de pescoço e punhos/mãos $(6,2 \%)$ e aumentaram nos cotovelos (de $0 \%$ para 6,2\%) entre os períodos estudados. Os dados podem ser observados na Tabela 3 juntos com a média dos intervalos e o desvio padrão.

Tabela 3: Queixas durante as atividades laborativas, domésticas e de lazer realizadas pelos funcionários do GE.

Regiões corporais

Pescoço
Ombros
Parte superior das costas

antes

$\begin{array}{ll}1 & 6,2 \\ 2 & 12,5 \\ 3 & 18,7\end{array}$

depois média dos intervalos

\begin{tabular}{l|l|l|l}
$\mathbf{0}$ & $\mathbf{N}$ & $\mathbf{0}$ & $\mathbf{0}$ \\
6,2 & 1 & 6,2 & 6,2 \\
12,5 & 1 & 6,2 & 9,3 \\
18,7 & 2 & 12,5 & 15,6
\end{tabular}




\begin{tabular}{llllll} 
Cotovelos & 0 & 00,0 & 1 & 6,2 & 3,1 \\
Punhos/mãos & 1 & 6,2 & 1 & 6,2 & 6,2 \\
Parte inferior das costas & 5 & 31,2 & 3 & 18,7 & 24,9 \\
Quadril/coxas & 1 & 6,2 & 0 & 00,0 & 3,1 \\
Joelhos & 2 & 12,5 & 1 & 6,2 & 9,3 \\
Tornozelos/pés & 1 & 6,2 & 0 & 00,0 & 3,1 \\
Média & & 11,0 & & 6,9 & 8,9 \\
DP & 9,2 & & 5,7 & 7,2 \\
\hline
\end{tabular}

${ }^{\star} \mathrm{DP}$ - Desvio Padrão

Já no GC, foi possível observar o aumento dos relatos de queixa para realização das atividades laborativas, domésticas e de lazer entre os períodos estudados nos segmentos de ombros (de $0 \%$ para 6,6\%); parte inferior das costas (de 6,6\% para 13,3\%); joelhos (de 6,6\% para 13,3\%). As queixas nos segmentos de punhos/mãos mantiveram-se (6,6\%). Não houve relatos de queixas nos segmentos de pescoço, parte superior das costas, cotovelos, quadril/coxa e tornozelos/pés entre os períodos estudados. Os dados podem ser observados na Tabela 4 juntos com a média dos intervalos e o desvio padrão.

Tabela 4: Queixas durante as atividades laborativas, domésticas e de lazer realizadas pelos funcionários do GC.

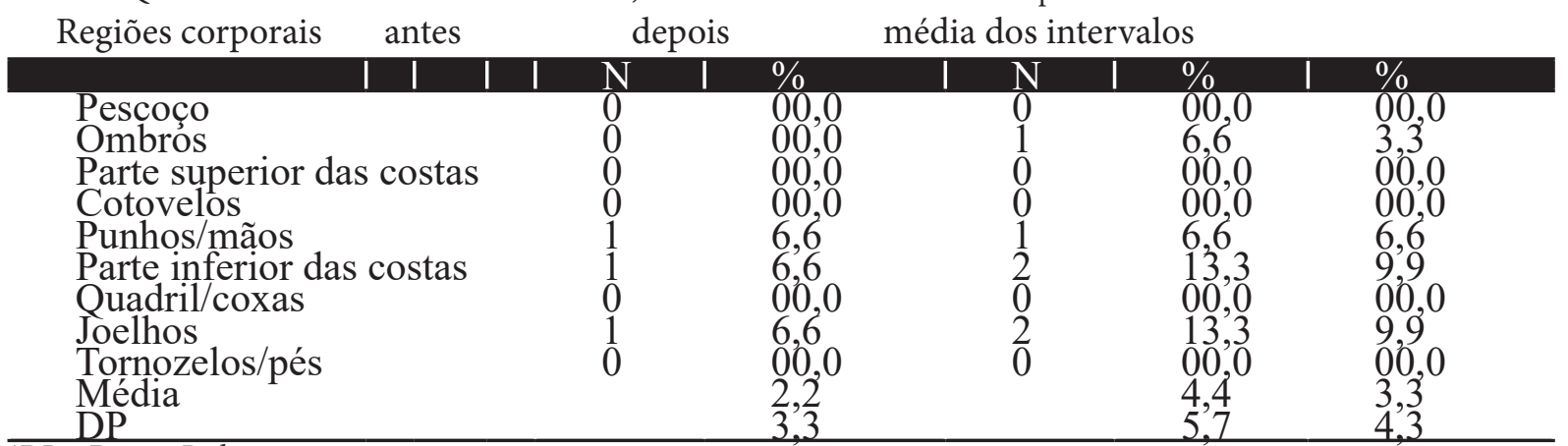

${ }^{*}$ DP - Desvio Padrão

Observou-se no GE entre os períodos antes e após 16 sessões de GL, redução dos relatos de consultas médicas e fisioterápicas por problemas nos segmentos de ombros (de $12,5 \%$ para $6,2 \%$ ); parte superior das costas (de $31,2 \%$ para $25,0 \%$ ); punhos/mãos (de 12,5\% para 6,2\%); parte inferior das costas (de $31,2 \%$ para $18,7 \%$ ); quadril/coxas (de 12,5\% para 6,2\%). Mesmo após a realização da GL as queixas mantiveram-se nos segmentos de pescoço $(12,5 \%)$ e joelhos $(6,2 \%)$. Não houve relatos de queixas nos segmentos de cotovelos e tornozelos/pés. Os dados podem ser observados na Tabela 5 juntos com a média dos intervalos e o desvio padrão.

Tabela 5: A procura por algum profissional da saúde pelos funcionários do GE.

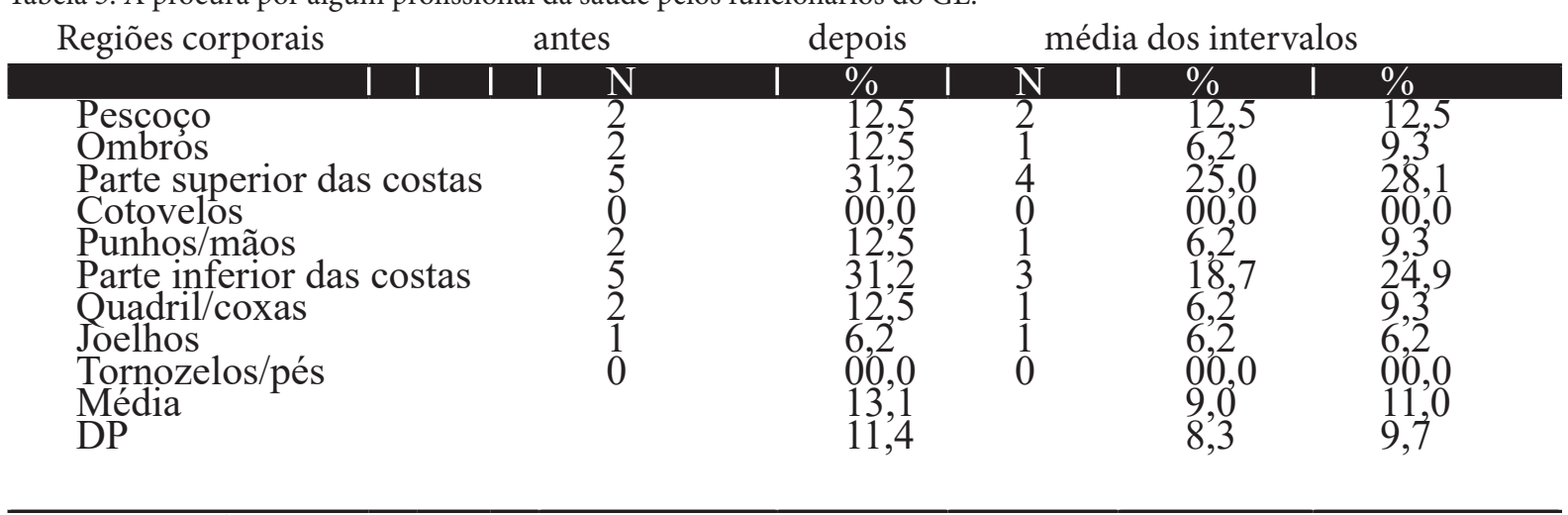

${ }^{{ }^{D} \mathrm{DP}}$ - Desvio Padrão 
No GC, também foi possível observar entre os períodos estudados redução dos relatos de consultas médicas e fisioterápicas por problemas nos segmentos de pescoço (de 13,3\% para 0,0\%); parte inferior das costas (de 20,0\% para 13,3\%); punhos/ mãos (de 13,3\% para 6,6\%); joelhos (de 20,0\% para
$6,6 \%$ ) e tornozelos/pés (de 6,6\% para $0 \%$ ). Houve aumento das queixas nos segmentos de ombros (de $0,0 \%$ para $6,6 \%$ ), porém não houve relatos na parte superior das costas; cotovelos e quadril/coxas. Os dados podem ser observados na Tabela 6 juntos com a média dos intervalos e o desvio padrão.

Tabela 6: A procura por algum profissional da saúde pelos funcionários do GC.

Regiões corporais antes

depois

média dos intervalos

\begin{tabular}{|c|c|c|c|c|c|}
\hline & $\mathrm{N}$ & $\%$ & $\mathrm{~N}$ & $\%$ & $\%$ \\
\hline Pescoço & 2 & 13,3 & 0 & 00,0 & 6,6 \\
\hline Ombros & 0 & 00,0 & 1 & 6,6 & 3,3 \\
\hline Parte superior das costas & 0 & 00,0 & 0 & 00,0 & 00,0 \\
\hline Cotovelos & 0 & 00,0 & 0 & 00,0 & 00,0 \\
\hline Punhos/mãos & 2 & 13,3 & 1 & 6,6 & 9,9 \\
\hline Parte inferior das costas & 3 & 20,0 & 2 & 13,3 & 16,6 \\
\hline Quadril/coxas & 0 & 00,0 & 0 & 00,0 & 00,0 \\
\hline Joelhos & 3 & 20,0 & 1 & 6,6 & 13,3 \\
\hline Tornozelos/pés & 1 & 6,6 & 0 & 00,0 & 3,3 \\
\hline Média & & 8,1 & & 3,6 & 5,8 \\
\hline DP & & 8,6 & & 4,8 & 6,1 \\
\hline
\end{tabular}

${ }^{\text {ॠDP - Desvio Padrão }}$

No GE, entre os períodos antes e após 16 sessões de GL, houve diminuição de algum problema nos segmentos de: pescoço (de $18,7 \%$ para $6,2 \%$ ); parte superior das costas (de $18,7 \%$ para $6,2 \%$ ); parte inferior das costas (de 25,0\% para 12,5\%) e quadril/coxas (de 18,7\% para 6,2\%). Mesmo após a realização da GL as queixas mantiveram-se nos segmentos de joelhos $(6,2 \%)$ e aumentaram nos ombros $(0 \%$ para $6,2 \%)$ e cotovelos $(0 \%$ para $6,2 \%)$. Não houve relatos de queixas nos segmentos de punhos/mãos e tornozelos/pés entre os períodos estudados. A média dos intervalos, média total e desvio padrão podem ser observados na Tabela 7.

Tabela 7: Ocorrência de algum problema nas regiões corporais dos funcionários do GE.

Regiões corporais antes de-
pois média dos intervalos

\begin{tabular}{|c|c|c|c|c|c|}
\hline & $\mathbf{N}$ & $\%$ & $\mathrm{~N}$ & $\%$ & $\%$ \\
\hline Pescoço & 3 & 18,7 & 1 & 6,2 & 12,4 \\
\hline Ombros & 0 & 00,0 & 1 & 6,2 & 3,1 \\
\hline Parte superior das costas & 3 & 18,7 & 1 & 6,2 & 12,4 \\
\hline Cotovelos & 0 & 00,0 & 1 & 6,2 & 3,1 \\
\hline Punhos/mãos & 0 & 00,0 & 0 & 00,0 & 00,0 \\
\hline Parte inferior das costas & 4 & 25,0 & 2 & 12,5 & 18,7 \\
\hline Quadril/coxas & 3 & 18,7 & 1 & 6,2 & 12,4 \\
\hline Joelhos & 1 & 6,2 & 1 & 6,2 & 3,1 \\
\hline Tornozelos/pés & 0 & 00,0 & 0 & 00,0 & 00,0 \\
\hline Média & & 9,7 & & 5,5 & 7,2 \\
\hline DP & & 10,4 & & 3,7 & 6,7 \\
\hline
\end{tabular}

${ }^{\star D P}$ - Desvio Padrão

No GC, entre os períodos antes e após 16 sessões de GL, observou-se aumento de algum problema nos segmentos de: pescoço $(26,6 \%$ para $33,3 \%)$; parte superior das costas $(0,0 \%$ para $26,6 \%)$; parte inferior das costas $(20,0 \%$ para $33,3 \%)$; joelhos $(13,3 \%$ para $33,3 \%)$ e tornozelos/pés (13,3\% para 26,6\%). As queixas mantiveram-se nos segmentos de ombros $(20,0 \%)$; punhos/mãos (33,3\%); quadril/ 
coxas $(13,3 \%)$ e diminuíram nos cotovelos (de $6,6 \%$ para $0,0 \%$ ). A média dos inter- valos, média total e desvio padrão podem ser observados na Tabela 8 .

Tabela 8: Ocorrência de algum problema nas regiões corporais dos funcionários do GC.

\begin{tabular}{|c|c|c|c|c|c|}
\hline & $\mathrm{N}$ & $\%$ & $\mathrm{~N}$ & $\%$ & $\%$ \\
\hline Pescoço & 4 & 26,6 & 5 & 33,3 & 29,9 \\
\hline Ombros & 3 & 20,0 & 3 & 20,0 & 20,0 \\
\hline Parte superior das costas & 0 & 00,0 & 4 & 26,6 & 13,3 \\
\hline Cotovelos & 1 & 6,6 & 0 & 00,0 & 3,3 \\
\hline Punhos/mãos & 5 & 33,3 & 5 & 33,3 & 33,3 \\
\hline Parte inferior das costas & 3 & 20,0 & 5 & 33,3 & 26,6 \\
\hline Quadril/coxas & 2 & 13,3 & 2 & 13,3 & 13,3 \\
\hline Joelhos & 2 & 13,3 & 5 & 33,3 & 23,3 \\
\hline Tornozelos/pés & 2 & 13,3 & 4 & 26,6 & 19,9 \\
\hline Média & & 16,2 & & 24,4 & 20,3 \\
\hline DP & & 10,0 & & 11,5 & 9,3 \\
\hline
\end{tabular}

${ }^{\star}$ DP - Desvio Padrão

\section{DISCUSSÃO}

Após a realização do protocolo de GL foi possível observar através do Questionário Nórdico de Sintomas Osteomusculares, diminuição dos sintomas de dor/formigamento no GE, principalmente nas regiões de ombros; parte superior das costas; punhos e mãos e quadril/coxas. A eficácia da GL compensatória em trabalhadores foi investigada, visando à redução do estresse ocupacional e da dor osteomuscular por Freitas-Swerts e Robazzi $(2014)^{12}$,cuja pesquisa constou de GL com frequência de duas vezes semanais, duração de 15 minutos cada, no período de 10 semanas. Constataram a presença de estresse ocupacional nos trabalhadores avaliados, entretanto, sem redução estatisticamente significativa dos escores após a GL. Contudo, houve redução álgica estatisticamente significativa nas regiões do pescoço, cervical, partes superiores, médias e inferiores das costas, coxa direita, perna esquerda, tornozelo direito e pés. Concluíram que a GL pode promover redução significativa de quadro álgico na coluna vertebral; todavia, não promoveu redução significativa dos níveis de estresse ocupacional. A atual pesquisa não teve como objetivo verificar os níveis de estresse ocupacional, porém com relação à redução álgica nos segmentos corporais os resultados encontrados por estes autores corroboram com os obtidos nesta pesquisa.
Outra pesquisa obteve resultados semelhantes aos evidenciados neste estudo. Os pesquisadores verificaram o efeito da GL sobre a dor nas costas e sobre os hábitos posturais de trabalhadores que ficavam por longos períodos na posição sentada. Participaram 30 trabalhadores do setor administrativo, divididos em GC $(n=15)$ e GE $(n=15)$, sendo este submetido a sessões de GL durante três meses. Os resultados demonstraram que a GL proporcionou diminuição da intensidade e frequência da dor referida aos trabalhadores do GE, e mudança do hábito postural durante o trabalho, melhorando a postura sentada ${ }^{11}$. Em contrapartida no GC, formado por funcionários que não aderiram ao programa de GL, foi possível verificar após os períodos estudados, o aumento dos relatos de dor/formigamento principalmente no pescoço $(33,3 \%$ para $46,4 \%)$ e parte superior das costas (20,0\% para $46,4 \%)$.

Um estudo realizado por Melo et al. (2013) ${ }^{12}$ teve como objetivo conhecer o grau de incidência de Distúrbios Osteomusculares Relacionados ao Trabalho (DORT) (fadiga muscular, dormência, formigamento, diminuição da sensibilidade e de força) em trabalhadores do Instituto Nacional de Metrologia Qualidade e Tecnologia (INMETRO). Observaram que as regiões anatômicas mais acometidas por esses sintomas foram: lombar (45\%), ombro $(42 \%)$ e punho $(28 \%)$ e que o uso genera- 
lizado do computador pode constituir fatores que potencializam a adoção de posturas inadequadas durante a jornada de trabalho. Baseados na pesquisa de Melo et al. (2013) $)^{13}$, pode-se correlacionar a presença dos sintomas dor/formigamento nos segmentos dos joelhos, parte inferior das costas, punhos/mãos no GC e a manutenção dos mesmos no segmento dos cotovelos em ambos os grupos, com as tarefas desempenhadas pelos participantes do presente estudo, visto que os funcionários trabalhavam em um escritório e permaneciam por longas horas de trabalho sentados, manuseando o computador. Corbinet al. (2006) apud Grande et al. (2013) ${ }^{14}$ ressaltam que longas horas de trabalho sentado favorecem encurtamento muscular na região posterior da coxa e no íliopsoas, aumenta a probabilidade de sobrepeso e os fatores de risco de doenças cardiovasculares. Segundo Andrade $(2001)^{15}$, trabalhadores que permanecem sentados durante toda jornada de trabalho apresentam um estilo de vida sedentário e relevantes fatores de risco à saúde.

Mesmo os joelhos sendo o segmento corporal com maior incidência de dor no GC, foi possível observar após os períodos estudados, diminuição dos sintomas. A hipótese é que isto pode estar relacionado à questão de que os funcionários mesmo não aderidos ao programa de GL da atual pesquisa, periodicamente recebiam orientações pelos Educadores Físicos e Fisioterapeutas da empresa sobre assuntos referentes ao estilo de vida, fatores de risco à saúde e a importância da prática de atividade física. Grande et al. (2013) ${ }^{14}$ investigaram fatores determinantes na qualidade de vida de 190 trabalhadores, após três meses de programas de promoção à saúde do trabalhador. A empresa "A" recebeu a GL, cartazes com recomendações de saúde e qualidade de vida e software computacional; a empresa "B" recebeu GL; a empresa "C" teve cartazes com recomendações de saúde e qualidade de vida e software computacional; a empresa "D" foi o controle. Observaram que fatores como condição física, tabagismo e tempo prolongado na posição sentada interferem na qualidade de vida dos funcionários. Por esse motivo, ressaltaram sobre a importância do entendimento dos componentes do estilo de vida no ambiente de trabalho e que intervenções educativas e aconselhamentos individuais ou coletivos são efetivos na promoção à saúde do trabalhador.
No presente estudo após 16 sessões de GL observou-se no GE diminuição dos relatos de queixas na realização das atividades laborativas, domésticas e de lazer principalmente na parte inferior das costas (31,2\% para $18,7 \%)$ e desaparecimento dos sintomas nos segmentos de quadril/coxas e tornozelos/pés. Martins e Martins $(2000)^{16}$ realizaram um estudo com 90 funcionários públicos e observaram que após 27 sessões de GL, 63,3\% dos participantes não tiveram mais sintomas decorrentes das DORTs, $14,4 \%$ melhoraram e $1,1 \%$ dos casos os sintomas desapareceram, corroborando com os achados atuais. Em contrapartida, observou-se no GE a manutenção e aumento respectivamente, das queixas nos segmentos de pescoço $(6,2 \%)$ e punhos/mãos $(6,2 \%)$ e cotovelos ( $0 \%$ para $6,2 \%)$ respectivamente, mesmo após a GL. De acordo com os autores Marckay et al. (2000); Diniz e Junior (1998); Trelha et al. (2002) apud Melo et al. (2013) ${ }^{13}$, atualmente, a maioria das atividades são executadas com os membros superiores, e pelo fato de poucos ambientes de trabalho serem adequados para as posturas que exercem sobrecargas em grupamentos musculares relacionados à coluna vertebral, este segmento e os membros superiores são comumente afetados pelas DORTs. Esses achados são ainda mais evidenciados quando comparado com o GC do presente estudo, onde foi possível observar que não houve relatos de queixas nos segmentos quadril/coxa e tornozelos/pés durante os períodos estudados, mas quando questionados sobre a região mais acometida predominou ombros $(0,0 \%$ para $6,6 \%)$, parte inferior das costas (6,6 \% para $13,3 \%)$ e joelhos (6,6\% para $13,3 \%)$.

Maciel, Coelho e Bacellar $(2015)^{17}$ realizaram uma pesquisa em 89 funcionários de uma empresa de confecções que permaneciam em postura estática por tempo prolongado. O estudo teve como objetivo conhecer dados epidemiológicos relativos a esta atividade laboral, tendo como instrumento de avaliação o Questionário Nórdico de Sintomas Osteomusculares. Observaram que em relação às regiões anatômicas, as maiores queixas foram em membros inferiores. Dos 80 funcionários que apresentaram sintomas musculoesqueléticos, 35\% relataram limitações para realizar atividades domésticas, trabalho e de lazer. Nota-se que o estudo de Maciel, Coelho e Bacellar (2015) ${ }^{17}$ se assemelha 
a atual pesquisa, pois as regiões com maiores sintomas musculoesqueléticos foram a coluna vertebral e os membros superiores. Ainda de acordo com Maciel, Fernandes e Medeiros (2006) ${ }^{18}$ é comum que um indivíduo relate vários locais acometidos simultaneamente, dependendo da atividade que desenvolve. Barreto; Grando (2001) ${ }^{19}$ e Pignati; Machado (2005) ${ }^{20}$ avaliaram funcionários de diferentes áreas de trabalho e observaram que a maior incidência de DORTs eram nas regiões de coluna lombar e membros superiores onde a maioria dos sintomas manifestaram-se nos membros superiores de forma crônica semelhando-se ao presente estudo.

Pedrozo e Parizotto (2015) ${ }^{21}$ realizaram um estudo para verificar a procura por atendimento médico devido às dores musculares de 42 comerciários de três grandes empresas comerciais após intervenção de um programa de GL. O estudo demonstrou significativa melhora com a intervenção $(\mathrm{p}=0,05)$, já que os relatos foram de diminuição de procura médica acreditando-se que tenha havido diminuição nas dores corporais. Souza e Júnior $(2004)^{22}$ demostraram em sua pesquisa que durante a implantação do programa de GL específico para trabalhadores em uma empresa de construção e montagem industrial houve a diminuição de atendimentos nos ambulatórios por queixas osteomusculares. Estes estudos corroboram com a presente pesquisa, pois foi possível verificar que após a GL houve redução dos relatos de consultas médicas e fisioterápicas principalmente por queixas osteomusculares na maioria dos segmentos avaliados no GE.

Souza e Júnior $(2004)^{22}$ relatam que a diminuição do absenteísmo e a ausência no trabalho com apresentação de atestados médicos, diminuiu bastante durante sua intervenção com o programa de GL. Ainda em relação ao absenteísmo, Mendes (2000) apud Silveira et al. $(2007)^{23}$ realizaram um levantamento das empresas que haviam implantado GL e os resultados e benefícios para a indústria e funcionários. Em relação aos benefícios da GL, $17 \%$ das empresas informaram que houve diminuição do absenteísmo e, em 33\%, diminuíram-se as doenças ocupacionais. $\mathrm{Na}$ presente pesquisa não foi avaliado o índice do absenteísmo no trabalho, todavia, através dos achados citados anteriormente, reafirma-se não só a importância da influ- ência da GL sobre os distúrbios osteomusculares dos trabalhadores, mas também na redução dos gastos das empresas com relação ao afastamento dos trabalhadores por doenças ocupacionais.

Bielemann, et al. (2010) apud Laux et al. $(2016)^{24}$ ressaltam o exercício físico como meio de prevenção de doenças, afirmando que sua prática diminui doenças crônicas, gerando uma economia nos gastos públicos da saúde. Reis et al. (2003) apud Laux et al. $(2016)^{24} \mathrm{em}$ seu estudo observaram uma diminuição dos atestados médicos, que tratavam especificamente sobre lombalgias, após a implantação do programa de GL, observaram diminuição das dores lombares com o decréscimo de $90 \%$ dos casos após a aplicação da GL. Fato que corrobora com os resultados encontrados nesse estudo, onde se verificou a redução de consultas médicas no GE principalmente por problemas na parte inferior das costas (31,2\% para $18,7 \%)$.

Cesaro e Magro (2012) ${ }^{25}$ analisaram as demandas e as ações de assistência à saúde do trabalhador em um serviço de Atenção Básica por meio das consultas médicas. A principal queixa apresentada pelos trabalhadores usuários foi dor osteomuscular, $45 \%$ dos participantes mencionaram como queixa principal as dores osteomusculares localizadas em diferentes regiões do corpo como membros inferiores, superiores e coluna cervical.

$\mathrm{Na}$ presente pesquisa foi possível observar no GC que também houve redução das consultas aos profissionais de saúde por problemas em alguns segmentos corporais, como pescoço (3,3\% para $0,0 \%)$ e joelhos (20,0\% para $6,6 \%)$, mesmo não estando aderidos ao programa de GL proposto. Hipoteticamente isto pode ter ocorrido pelo fato de que os funcionários tinham um programa de palestras periódicas oferecidas pela empresa sobre os assuntos referentes ao estilo de vida, fatores de risco à saúde e a importância da prática de atividade física o que nos leva a supor que esses funcionários poderiam ter incluído a prática de atividade física em seu lazer, ou seja, fora do seu setor de trabalho. Militão (2001) ${ }^{26}$ após uma pesquisa observou que $55,5 \%$ dos funcionários sentiram-se mais motivados à prática de exercícios e $37 \%$ tiveram mudanças no lazer, passando a praticar esportes no final de semana após orientações de facilitadores e professores de Educação Física sobre a influência da atividade física na saúde do trabalhador. 
Lopes, Nogueira e Martinez (2008) ${ }^{27}$ investigaram a procura de auxílio médico de trabalhadores com sintomas osteomusculares provocados pelas atividades laborais. Observaram que os ombros, cotovelos e punhos, foram os segmentos mais acometidos, corroborando com a presente pesquisa onde houve aumento das queixas no segmento dos ombros do GC, motivo por procura de auxílio médico após os períodos estudados.

Em uma investigação realizada por Gurgueira et al. (2003) apud Picoloto e Silveira (2008) ${ }^{28}$ sobre a prevalência dos sintomas osteomusculares em trabalhadores, verificaram que as maiores queixas estavam relacionadas a região lombar considerando os últimos doze meses e últimos sete dias. Confirmando os dados encontrados no GE, no qual a região mais acometida e de maior melhora dos sintomas foi a parte inferior das costas $(25,0 \%$ para $12,5 \%$ ) nos últimos 7 dias.

Ainda Picoloto e Silveira $(2008)^{28}$ em um estudo com professores do ensino fundamental, observaram também o predomínio de problemas osteomusculares na região lombar $(63,1 \%)$, torácica $(62,4 \%)$ e cervical $(59,2 \%)$ nos últimos doze meses; porém, nos últimos sete dias, a sintomatologia foi predominante nos ombros $(29,9 \%)$, cervical $(28,7 \%)$ e lombar $(27,4 \%)$, corroborando com os resultados da reaplicação do questionário do GC do presente estudo. Nesta reavaliação da sintomatologia dos funcionários do GC, pode ser observada também uma redução das queixas nos cotovelos $(6,6 \%$ para $0,0 \%)$, supõe-se que isso foi possível, pois os trabalhadores podem se adaptar a dor, aprendendo a conviver com ela e realizando a automedicação, conforme foi demonstrado em uma pesquisa onde $23 \%$ da amostra relatou a utilização de analgésicos para que conseguissem realizar suas atividades e descansar após o trabalho.

Barcelos $(2000)^{29}$ afirma que a GL pode auxiliar na percepção corporal, induzindo os funcionários a assumirem posturas mais adequadas. Santos $(2000)^{30}$ observou no final de sua pesquisa que os funcionários de um supermercado do Sul do Brasil após serem submetidos ao um programa de GL relataram melhor disposição para o trabalho, diminuição do cansaço físico e das dores no final do dia; redução do estresse e alívio das tensões; melhoria da saúde física e mental. Santos $(2000)^{30}$ ainda afirma que a GL pode proporcionar benefícios físicos e mentais aos trabalhadores e assim também trazer benefícios para as empresas como aumento da produtividade; diminuição de incidência de doenças ocupacionais; menores gastos com despesas médicas; redução do índice de absenteísmo e rotatividade dos funcionários. Com os dados evidenciados no presente estudo, foi possível verificar que a GL pode promover melhora dos sintomas osteomusculares nos trabalhadores aderidos ao programa.

\section{CONCLUSÃO}

Com a realização da pesquisa foi possível verificar que a GL pode promover efeitos benéficos sobre os distúrbios osteomusculares de funcionários de uma empresa do ramo siderúrgico, diminuindo os relatos de queixas de dor/formigamento para a realização das atividades laborativas, domésticas e de lazer, bem como das consultas com algum profissional da saúde.

As regiões mais acometidas foram a parte superior e inferior das costas no grupo de trabalhadores aderidos ao programa de GL associando isto ao perfil dos funcionários avaliados que trabalhavam por horas prolongadas na posição sentada em um escritório. Conforme verificado no GC, supõe-se que palestras sobre estilo de vida, fatores de risco à saúde e a importância da prática de atividade física possam ser recursos também eficazes para a saúde do trabalhador, hipótese que poderá servir de análise para futuros estudos. A GL deve ser entendida como mais uma ferramenta de prevenção para a saúde e bem-estar do trabalhador, mas é importante ressaltar a necessidade de avaliar não só apenas os distúrbios osteomusculares em trabalhadores sendo relevante associar a influência das barreiras ergonômicas no desenvolvimento dos mesmos.

Dessa forma sugere-se a necessidade de outros estudos, em diferentes setores da indústria e em diferentes populações de trabalhadores, assim complementando esses achados.

\section{REFERÊNCIAS}

1. Mezzomo SP, Contreira AR, Corazza ST. Os efeitos da Ginástica Laboral sobre as habilidades básicas de funcionários de setores administrativos. Rev. Bras. Ciênc. Saúde. 2010; 8(25):6-13. 
2. Brasil, Ministério da Saúde. Protocolo de investigação, diagnóstico, tratamento e prevenção de lesões por esforços repetitivos: LER/DORT distúrbios osteomusculares relacionados ao trabalho. Brasília: Ministério da Saúde. 2000.

3. Neves JAC, Maduro PA, Mesquita FOS, Souza FT, Silva TFA. Distúrbios musculoesqueléticos autorreferidos em assistentes administrativos do Hospital Universitário de Petrolina, Pernambuco. Arq. em Mov. 2017;13(1):32-42.

4. Sampaio AA, Oliveira JRG. A ginástica laboral na promoção da saúde e melhoria da qualidade de vida no trabalho. Cad. de Ed. Fís. 2008;7(13):71-79.

5. Serra MVGB, Pimenta LC, Quemelo PRV. Efeitos da ginástica laboral na saúde do trabalhador: uma revisão da literatura. Rev. Pesq. em Fisioter. 2014;4(3):197-205.

6. Neves PA. Uma revisão bibliográfica sobre a importância da motivação na ginástica laboral. Trabalho de Conclusão de Curso. Porto Alegre: Universidade Federal do Rio Grande do Sul; 2015.

7. Freitas FCT, Swerts OSD, Robazzi MLCC. A ginástica laboral como objeto de estudo. Fisioter. Bras. 2009;10(5):364-370.

8. Silva CAR, Teodoro DCRC, Mendonça MRC, Alves AG, Alves FAVB, Araújo RF, Nogueira MS, Villar AC. Efeitos da ginástica laboral na qualidade de vida de trabalhadores da cerâmica primos de Adelândia- GO. Revista Eletrônica FMB. 2016;8(3):125-179.

9. Sadir MA, Bignotto MM, Lipp MEN. Stress e qualidade de vida: influência de algumas variáveis pessoais. Paidéia. 2010;20(45):73-81.

10. Barbosa PH, Carneiro F, Delmim LR, Hunger MS, Martelli A. Doenças osteomusculares relacionadas ao trabalho e à ginástica laboral como estratégia de enfrentamento. Arch. Health Invest. 2017;3(5):57-65.

11. Kuorinka I, Jonsson B, Kilbom A, Vinterberg H, Biering-Sorensen F, Andersson G, Jorgensen K. Standardised Nordic questionnaires for the analysis of musculoskeletal symptoms. Appl. Ergon. 1987;18(3):233-237.

12. Freitas-Swerts FCT, Robazzi MLCC. Efeitos da ginástica laboral compensatória na redução do estresse ocupacional e dor osteomuscular. Rev. Latino-Am Enfermagem. 2014; 22(4):629-36.

13. Candotti CT; Silva MR; Noll M; Lucchese CR. Efeito da ginástica laboral sobre a motivação para a prática regular de atividade física. Rev. Baiana de Saúde Pública. 2012;35(2):485-497.

14. Melo VF, Barros IM, Freitas NAB, Luzes R. Incidência de distúrbios osteomusculares relacionados ao trabalho (DORT), em trabalhadores do setor administrativo do instituto nacional de metrologia, qualidade e tecnologia (INMETRO). Rev. Saúde Física \& Mental. 2013;2(1):22-29.

15. Grande AJ, Silva V, Manzatto L, Rocha TBX, Martins GC, Júnior GBV. Determinantes da qualidade de vida no trabalho: ensaio clínico controlado e randomizado por clusters. Rev. Bras. Med. Esp. 2013;19(5):371-375.
16. Andrade, A. Ocorrência e controle subjetivo do stress na percepção de bancários ativos e sedentários: a importância do sujeito na relação atividade física e saúde [tese]. Florianópolis: Universidade Federal de Santa Catarina; 2001.

17. Martins CO, Martins, MO. Eficácia da ginástica laboral na prevenção aos DORT e sua aceitação por funcionários públicos de Florianópolis - SC. Anais do XXIII Simpósio Internacional de Ciências do Esporte - Atividade Física, Fitness e Esporte. 2000: 173.

18. Maciel RRBT, Coelho DS, Bacellar RB. Prevalência de sintomas musculoesqueléticos em trabalhadores de uma empresa de confecção em Salvador - Bahia. Ciência (In) Cena Bahia. 2015;(2):28-43.

19. Maciel ACC, Fernandes MB, Medeiros LS. Prevalência e fatores associados à sintomatologia dolorosa entre profissionais da indústria têxtil. Rev. Bras. de Epidemiol. 2006;9(1):94-102.

20. Barreto SJ, Grando K. Estudo sobre a importância da ergomotricidade na prevenção de doenças ocupacionais em servidores da universidade regional de Blumenau. In: $5^{\circ}$ Congresso Paulista de Educação Física, 2001, Jundiaí. Anais do $5^{\circ}$ Congresso Paulista de Educação Física. p. 87-87.

21. Pignati WA, Machado JMH. Riscos e agravos à saúde e à vida dos trabalhadores das indústrias madeireiras de Mato Grosso. Rev. Ciên. \& Saúde Colet. 2005;10(4):961-973.

22. Pedroso WR, Parizotto ZAM. Ginástica laboral: as perspectivas da sua prática na redução do cansaço físico. FIEP Bulletin on-line. 2015;85.

23. Souza I, Júnior RV. Ginástica laboral: contribuições para a saúde e qualidade de vida de trabalhadores da indústria de construção e montagem - Case TECHINT S.A. Rev. Digital. 2004;77(10):01-18.

24. Silveira MG, Silva RP, Reis VMMR, Novaes JS. Efeitos da ginástica laboral nas variáveis morfológicas, funcionais, estilo de vida e absenteísmo dos trabalhadores da indústria farmacêutica de Montes Claros/MG. Fitness \& Performance Journal. 2007;6(5):295-301.

25. Laux RC, Pagliari P, Junior JVE, Corazza ST. Programa de Ginástica Laboral e a Redução de Atestados Médicos. Ciênc \& Trab. 2016;18(56):130-133.

26. Cesaro F, Magro MLPD. A atenção à saúde do trabalhador no âmbito da atenção básica: um estudo de caso. Rev. Grifos. 2012;21(33):149-169.

27. Militão AG. A influência da ginástica laboral para a saúde dos trabalhadores e sua relação com os profissionais que a orientam [tese]. Florianópolis: Universidade Federal de Santa Catarina; 2001.

28. Lopes ECT, Nogueira JE, Martinez LRM. Influência da ginástica laboral em funcionários que trabalham com telemarketing. Rev. Digital-Buenos Aires. 2008;13(124):01-11.

29. Picoloto D; Silveira E. Prevalência de sintomas osteomusculares e fatores associados em trabalhadores de uma indústria metalúrgica de Canoas - RS. Ciên.Saúde Colet. 2008;13(2):507-516. 
30. Barcelos RC. Acompanhamento de um programa de exercícios laborais nos funcionários do SENAI no departamento Regional de Santa Catarina: um estudo de caso. Monografia (Conclusão do curso de Educação Física). CEFID/UDESC, Florianópolis, 2000.

31. Santos LM. Benefícios da ginástica laboral e sua influência sobre o índice de absenteísmo. Monografia (Conclusão de curso de Licenciatura em Educação Física). CEFID/UDESC. Florianópolis, 2000.

Como citar este artigo:

Souza JR, Martins MT, Souza CM, Baptista IC, Cardoso LP, Medeiros GB, Cruz AT, Januário PO. A influência da ginástica laboral nos distúrbios osteomusculares relacionados ao trabalho. Rev. Aten. Saúde. 2018;16(58):63-74. 